\title{
BMJ Open Smoking cessation interventions in South Asian countries: protocol for scoping review
}

\author{
Sajid lqbal (D) , Rubina Barolia, Laila Ladak, Pammla Petrucka
}

To cite: Iqbal S, Barolia R, Ladak L, et al. Smoking cessation interventions in South Asian countries: protocol for scoping review. BMJ Open 2021;11:e038818. doi:10.1136/ bmjopen-2020-038818

- Prepublication history and supplemental material for this paper is available online. To view these files, please visit the journal online (http://dx.doi org/10.1136/bmjopen-2020038818).

Received 02 April 2020 Revised 28 December 2020 Accepted 07 January 2021

\section{Check for updates}

(c) Author(s) (or their employer(s)) 2021. Re-use permitted under CC BY-NC. No commercial re-use. See rights and permissions. Published by BMJ.

Aga Khan University School of Nursing and Midwifery, Karachi, Pakistan

Correspondence to

Mr Sajid lqbal;

sajidchakesar@hotmail.com

\begin{abstract}
Introduction Unfortunately, like many other health risks, smoking rate has been on the rise in developing countries. Half of current smokers in the world reside in only three countries of Asia that include India. Many smoking cessation interventions that were developed and successfully implemented in the context of developed countries have not been equally successful in South Asia. Hence, there is a dire need of culturally relevant smoking cessation interventions. We propose a scoping review with objectives to explore the extent and nature of interventions for smoking cessation and its associated factors in South Asian Region by systematically reviewing the available published and unpublished literature.
\end{abstract}

Methods and analysis The review has been registered in Joanna Briggs Institute (JBI) systematic reviews register and details are given in the Methodology section. The updated framework of JBI for scoping review methodology will be used as guide for conduct of this scoping review. Electronic databases (PubMed, CINAHL plus, Proquest Theses and Dissertations, EBSCO Dentistry and Oral Sciences, and Wiley Cochrane Library), reference lists of selected studies and grey literature will be considered for inclusion in this review. The literature search is anticipated to be carried out in December-January 2020. Initially, two reviewers in consultation with a librarian will develop search syntax followed by search from the selected sources. Consequently, the reviewers will screen all the titles, abstracts and full articles to establish relevance of each study for inclusion. Factors associated with smoking cessation will be coded and categorised applying qualitative content analysis, while interventions extracted from the literature will be described with the stated level of effectiveness.

\section{INTRODUCTION}

Globally, in a year over 6 million people die due to causes related to tobacco use. This number is anticipated to reach approximately 8.3 million/year by 2030 , primarily due to increasing prevalence of tobacco use in low-income and middle-income countries. ${ }^{12}$ The increasing prevalence of cigarette smoking and its hazardous consequences are documented extensively. It is estimated that, globally, 942 million men and 175 million women over age 15 years are daily smokers; consequently, cigarette smoking is the single

\section{Strengths and limitations of this study}

- The review includes content in any language with English translation available and search in three different ways from South Asian Region (SAR), thus, increasing the chances of covering a broader range of work done on phenomena of interest.

- Most of the team members in this review are experts of systematic reviews who will ensure each step carried out with utmost accuracy.

- One of the strengths of this review is engagement of stakeholders and relevant organisations during the review process.

- The quality of included studies will not be ensured through an established tool, although some more recent scoping review protocols have used tools such as the Newcastle-0ttawa Scale.

- Literature published in languages other than English will not be included that may affect the scope of the review. However, preliminary search shows rare availability of literature in language other than English in SAR.

leading cause of preventable diseases and deaths making it a critical public health challenge. ${ }^{3}{ }^{4}$ Cigarette smoking leads to a range of health problems that either affect the quality of life or become the reason for premature death. Smoking tobacco exposes the smoker's body to over 7000 toxic chemicals that include a minimum of 70 carcinogens affecting almost every body system. ${ }^{3}$ Significant health problems due to smoking include cardiovascular (mainly coronary heart) diseases and respiratory diseases, mainly cancer. ${ }^{56}$ Moreover, smoking is a risk factor for congenital disorders in the case of pregnant smokers, stroke, peripheral vascular diseases, cerebrovascular disease, renal failure, intestinal ischaemia and impotence to name a few. ${ }^{3}$ Furthermore, although the consequences of smoking are largely associated with the number of cigarettes smoked, a lifelong regular smoker loses approximately 10 years of life to smoking. ${ }^{3}$ Of importance are the benefits of non-smoking or smoking 
cessation. Quitting before age 40 years saves about 9 years of life because cessation at this age reduces the risk of death due to smoking-related morbidities by $90 \% .{ }^{3} \mathrm{~A}$ smoker can also benefit from smoking cessation even after the development of smoking-related diseases such as chronic obstructive pulmonary diseases and cardiovascular diseases. $^{8}$

Hence, smoking is likely one of the most lethal and prevalent yet preventable health risk behaviours in today's world. Its increasing epidemic levels should catalyse the need for better smoking cessation measures.

\section{Definitions of terms}

Smoker

Smoker is defined as a person who smoked at least once daily in the last 30 days.

\section{Smoking cessation rate}

Smoking cessation rate (outcome measure) in this study is defined as the decrease in number of cigarettes per day in comparison with the pre-intervention phase.

\section{Smoking cessation intervention}

Any action intended to increase cigarette smoking cessation rate will be referred as smoking cessation intervention.

\section{South Asian Region}

South Asia is one of the most populated geographical regions inhabited by almost a quarter of the world's population. ${ }^{9}$ South Asian Region (SAR) includes Pakistan, India, Sri Lanka, Afghanistan, Bangladesh, Nepal, Bhutan and Maldives. ${ }^{10}$ Three of the countries (Pakistan, India and Bangladesh) mentioned in the above list are partition of one country, that is, British India, ${ }^{9}$ thus, sharing numerous cultural and religious traits. Besides, a common issue faced by people in countries of SAR is their lack of awareness of different health risks and low level of education. Sreeramareddy et al found that in two of the countries in SAR (ie, Bangladesh and Maldives), one in three men between 15 and 49 years is not educated, while $17.1 \%-56.2 \%$ women of aged $15-49$ years were uneducated. ${ }^{11}$ The study also emphasised on negative association of education level with smoking behaviour. ${ }^{11}$

Smoking is the most common health risk found high in South Asian population. ${ }^{12}$ A study on Asian population reports that serving tobacco products is a cultural norm and dignity of Asian people. ${ }^{12}$ Concurrently, the study by Sreeramareddy et al found the prevalence of smoking among men aged between 15 and 49 years as $60 \%$ in Bangladesh, $47.3 \%$ in Maldives, $34.1 \%$ in India, $33.6 \%$ in Nepal and $31.6 \%$ in Pakistan. ${ }^{11}$ The study also concluded that smoking in South and Southeast Asian countries is more common among men from low economic backgrounds, uneducated or residents of rural areas. ${ }^{11}$ Therefore, consideration of the context-specific factors associated with smoking is important in planning strategies for prevention and control of cigarette smoking.

\section{Study rationale}

Despite claims by different countries regarding progress towards achievement of their smoking cessation goals, globally the decline in smoking prevalence has been slower in the last 10 years (2005-2015) than in the past (1990-2005). ${ }^{13}$ In developed countries, efforts for smoking cessation remained quite effective due to factors like good public awareness, availability of resources for smoking cessation and uniform policies towards smoking cessation; whereas, in low-income and middleincome countries, the smoking cessation rate remained discouraging. ${ }^{14-16}$ Consequently, if the current situation sustains, as predicted, by 2030 , almost $80 \%$ of tobaccorelated deaths will be in low-income and middle-income countries. ${ }^{17}$

Although systematic reviews either based on data from one specific country ${ }^{18}$ or general global data ${ }^{19}$ have been carried out, no review of smoking cessation interventions in SAR countries has been conducted on smoking cessation and its associated factors. The strict inclusion criteria of previously conducted systematic reviews might have significantly reduced the inclusion of available data on the phenomena of interest. Therefore, a scoping review is proposed with primary outcome to summarise the current scope of interventions and critical factors to be considered while planning any smoking cessation intervention. This will help in achieving secondary outcome, that is, to enhance effectiveness of the developing strategies for reduction of smoking prevalence in SAR. Moreover, the increasing prevalence of smoking in SAR signifies the imperative for reconsideration of the strategies implemented for smoking cessation in this region. Therefore, it is important to explore the range and nature of these strategies to align them, through further research, with local contextual factors to potentiate achievement of optimal outcomes.

\section{Why a scoping review?}

Scoping review is an approach that covers underpinning concepts surrounding the phenomenon of interest ${ }^{20}$ thus, giving a broader image that serves as a precursor to systematic reviews. ${ }^{21}$ Also, scoping reviews give depth and breadth of the research work done on a topic, thus increasing the understanding of the nature of the phenomenon under investigation. ${ }^{22}$

Effective interventions to stop or control the current pattern of smoking are emerging and evolving constantly. Due to this variability and complexity, there is a need to collate evidence related to interventions for smoking cessation among South Asian population and distinguishing the underpinning forces that encourage or discourage the behaviour of smoking. Given the range of programmes, national initiatives/policies and potential interventions, it is desirable that the least restrictive design for a review be selected to maximise the diversity for smoking cessation strategies in SAR. We want to take into account the cultural specific measures or interventions which are not formally reported or not publicly 
available. Additionally, in this study we are not focusing on a clinical question which would more closely align with a systematic review, whereas, the scoping review is more aligned with mapping the evidence related to the types of smoking cessation interventions and the characteristics of individuals accessing such programmes. ${ }^{21}$ After consideration, a scoping review was deemed as best serving the aim of this study.

\section{METHODOLOGY AND ANALYSIS}

The title of this protocol has been registered with 'Joanna Briggs Institute (JBI) systematic review register' page 10, on 28 January 2020 and can be found on their website (https://jbi.global/systematic-review-register? combine $=\&$ \&items_per_page $=10 \&$ page $=17 \&$ corder $=$ title\& sort=asc).

Besides, the conduct and reporting of the scoping review report will be guided by the Preferred Reporting Items for Systematic Reviews and Meta-Analyses (PRISMA) extension for Scoping Review as recommended in literature. ${ }^{23}{ }^{24}$ Moreover, to advance the development process and reporting of this protocol, the recommended PRISMA-Protocols checklist was used ${ }^{25}$ (attached as online supplemental appendix 1). In addition, the record and data of the review will be saved in a computer and in a hard drive for back-up. Also, the computer files will be connected to campus cloud in order to secure the data.

This review will be part of a multiphasic mixed-method research to include contextually relevant smoking cessation interventions and associated factors that will help in the development of a model for smoking cessation in SAR. Since the review is from existing published and publicly available literature, it does not require an ethical approval. A tentative timeline for the conduction of the review is attached as online supplemental appendix 2.

\section{Patient and public involvement}

This review will not include any research participants or patients for data collection. The stakeholders' involvement in initial planning and research question setting will be acknowledged in the original report of the review as per their permission. Moreover, the findings of the proposed review will be disseminated to smokers and relevant stakeholders to encourage their involvement in further planning for smoking cessation.

\section{Protocol design}

The JBI guidelines proposed by Peters $e t a l^{24}$ underpinned by the framework presented by Arksey and O'Malley ${ }^{20}$ and Levac $e t a l^{26}$ will guide the scoping review to examine the extent and nature of work done on smoking cessation in SAR with the factors impacting these efforts. Thus, the review will be carried out in the nine stages mentioned herein. Also, we have experts in our team who have worked on topic of the scoping review. In addition to that, we will consult a smoking cessation consultant (JA) available in our university. The stated expertise will be instrumental in how to conduct an informed search, review and synthesis process.

\section{Stage 1: defining and aligning the objective/s and question/s}

The scoping review is planned to systematically search literature across the range and nature of interventions for smoking cessation and to map the factors associated with such interventions. Following this, questions for the proposed scoping review are designed as: what is known from existing literature about the range of interventions/ control measures devised for smoking cessation in SAR? and What are the factors associated with smoking cessation in SAR in the available literature?

\section{Stage 2: developing and aligning the inclusion criteria with the} objective/s and question/s

Aligned with the questions and objectives of the review, studies regardless of design including qualitative studies, randomised controlled trials, quasi-experimental studies, case-control studies, cohort studies, or descriptive studies addressing the phenomenon of smoking cessation or intervening smoking behaviour or factors associated with smoking cessation or combination of one or more of these phenomena will be included. The target population of the included studies will be adults (age above 18 years) from any country in SAR. Literature written in English or any other language with English translation available will be included. Furthermore, due to rich availability of data, studies published within 5 years from search date will be included.

Studies will be excluded if not available as free full text. Moreover, studies having participants from any country other than countries in SAR will not be included.

Stage 3: describing the planned approach to evidence searching, selection, data extraction and presentation of the evidence Initially, two reviewers will extensively search the selected databases (PubMed, CINAHL, Proquest Theses and Dissertations, EBSCO Dentistry and Oral Sciences, and Wiley Cochrane Library) for relevant qualitative and quantitative data on the phenomena of interest during the period mentioned in the timeline. PubMed is recommended for inclusion in systematic/scoping reviews as it covers an extensive range of literature. ${ }^{27}$ Moreover, most of the rest are health-related literature databases; therefore, their inclusion in the present scoping review can add significant data on preventing health hazards of smoking through different interventions. CINAHL, on the other hand, is a specialised database for nursing-related literature, ${ }^{27}$ thus adding to the phenomenon from nursing lens and broadening the range of the data beyond positivist view.

The anticipated dates for the literature search are mentioned in online supplemental appendix 2. The literature search will be carried out in the four steps discussed below in the Search strategy section. To maintain a rigorous record of the searched literature, the PRISMA flow diagram will be used. 


\begin{tabular}{|c|c|c|c|c|c|}
\hline Search & Actions & Details & Query & Results & Time \\
\hline$\# 7$ & $\cdots$ & $>$ & $\begin{array}{l}\text { Search: }(((\# 1) \text { AND (\#2)) AND (\#3)) AND (\#4) Filters: Full text, in the } \\
\text { last } 5 \text { years }\end{array}$ & 259 & 02:38:54 \\
\hline \#6 & $\cdots$ & $>$ & Search: (((\#1) AND (\#2)) AND (\#3)) AND (\#4) Filters: Full text & 558 & $02: 34: 57$ \\
\hline \#5 & $\cdots$ & $>$ & Search: (((\#1) AND (\#2)) AND (\#3)) AND (\#4) & 620 & 02:34:05 \\
\hline \#3 & $\cdots$ & $>$ & $\begin{array}{l}\text { Search: (("Smoking Cessation" [Mesh:NoExp]) OR (Cessation)) OR } \\
\text { (Quit*) }\end{array}$ & 183,644 & 02:24:04 \\
\hline
\end{tabular}

Figure 1 PubMed search conducted in November 2020.

\section{Stage 4: searching for the evidence}

\section{Step 1}

After a thorough discussion with research team and the librarian on the selection of appropriate keywords, controlled vocabulary and going through relevant literature, the following key terms supported by Medical Subject Headings (MeSH) terms, Boolean phrases and wildcards were considered for literature search: tobacco, cigarette, smoking cessation, quit, interventions, measures, strategies and South Asian Region. Consequently, a nested search syntax was developed as, (Intervention* OR measure* OR strateg* OR program) AND ("Smoking"[Mesh] OR smok* OR Tobacco OR Nicotine OR Cigarette) AND ("Smoking Cessation" [Mesh:NoExp] OR Cessation OR Quit*) AND ("South Asia*" OR Pakistan OR Afghanistan OR Bangladesh OR Bhutan OR India OR Nepal OR Maldives OR Sri Lanka). The filters applied included full-text articles and publications in the last 5 years. The mentioned key terms were used to develop full strategy for search in PubMed (attached as figure 1 in image).

\section{Step 2}

The reference list of selected articles will be manually checked and identified relevant references will be used for additional article retrieval through citation chaining. This will also assist in identifying relevant organisational websites in SAR.

\section{Step 3}

Hand searching of print journals will be carried out in order to capture the literature available in library that might have been missed while searching through selected search engines.

\section{Step 4}

Data received through coordination with other research colleagues or key informants, information printed in alternate formats (ie, reports, briefs), and any available unpublished material will be sought and reviewed for relevancy to the intent of this review. Unpublished literature search will be supported through email contacts with authors of published articles to share any source of grey literature. Moreover, we will access Proquest Theses and Dissertations Database, OpenGrey database and Higher Education Commission Research Repository. Also, we will use the WHO country reports on cigarette smoking.

\section{Stage 5: selecting the evidence}

Based on relevance, accessed studies will be considered for inclusion through EndNote software. Initially, two reviewers will independently read the title and abstract of a retrieved study. Based on relevancy, the reviewers will recommend, include or exclude. Then, individually, the reviewers will read the included full-length articles and independently rate the appropriateness of the article or any reading material for inclusion. The reviewers will then cross check the list of included and excluded studies. If any discordance occurs respecting inclusion or exclusion of any full-text article or any other material (eg, reports, brief and so on) selected at the preliminary stage, the article or other material will be reread; and if still not clear to include or exclude, a third reviewer will be involved to build consensus. The appropriateness of the selected material will be based on inclusion criteria and authenticity of the publishing authority or of the organisation to which unpublished work is associated.

\section{Stage 6: extracting the evidence}

Data will be collected using a study instrument developed by researchers that include, but not limited to, study reference, location, participants' characteristics (ie, age, gender and ethnicity), methodology, research purpose, data collection and main relevant outcomes. This analytical form will be pretested and discussed with 
research team to ensure it is capturing all relevant information (numerical data and narrative accounts) from a selected study. Quality assessment of included literature will not be performed as the intent of scoping review is to examine the breadth and depth of a phenomenon and not to check the robustness of the selected studies. ${ }^{20}{ }^{28} \mathrm{At}$ this stage, both the reviewers will share the extracted data sheets filled from the included articles.

\section{Stage 7: analysis of the evidence}

The scoping reviews aim to develop a map of the underpinning concepts of the selected research area, the formulation of results leads to the range of work being done. ${ }^{27}$ The proposed review will extract the extent and nature of work done for smoking cessation as well as the range of factors that might have affected such work. Furthermore, the review will not weigh or assess the impact of the interventions on smoking cessation as they all will be taken into consideration to further guide the linear analysis process described here. In the proposed scoping review, the extracted interventions will be coded based on the Framework Convention on Tobacco Control set forth by the $\mathrm{WHO},{ }^{29}$ and the smoking cessation-associated factors will be coded thematically. All the factors that have been described in selected studies, reports or briefings will be colour coded. Based on the nature of codes, they will be put under relevant categories, such as personal factors, social factors and so forth. Finally, according to the focus of categories, themes will be assigned to make it more comprehensible.

To clarify the results, quantitative and qualitative studies will be synthesised separately. First, individually, both reviewers will summarise findings from all included studies. Second, the reviewers will discuss their findings and will merge them. Findings will then be shared with the whole research team including the supervisor of the study.

To minimise bias arising from personal values, preconceptions and judgements, the reviewers will undertake bracketing before data collection. The review process will involve two or more members of the research team in conducting the reviews in order to ensure capturing/ revealing multiple perspectives. Moreover, reviewers will maintain a diary to capture their feelings and learning experience in real time. In order to keep an organised and detailed record of reflexive bracketing, an iterative process of reflexive journey will be maintained throughout the review process. ${ }^{30}$ Through these strategies, we believe reflexivity will be achieved.

\section{Stage 8: presentation of the results}

The findings of this review will be presented in tables, appropriate diagrams and texts. The findings of the review will be shared through publications, posters, conference presentations and meetings with different stakeholders.
Stage 9: summarising the evidence in relation to the purpose of the review, making conclusions and noting any implications of the findings

Based on the findings, inferences will be drawn for presentation of the whole scenario with clarity and brevity. Moreover, recommendations will be set forth to make effective use of the findings in future planning for smoking cessation in SAR.

\section{Ethics and dissemination}

The study does not require an ethical approval. However, every effort will be put to maintain transparency in the conduct of the study. The results of this scoping review will be disseminated through publications, presentations in conferences and meetings with stakeholders.

Contributors SI formatted and outlined the protocol, and conducted frequent meetings with the librarian and reviewers to finalise the included databases, frameworks, and inclusion and exclusion criteria. He wrote the protocol and is the guarantor of this review. RB came up with the initial idea of the review, supervised the whole activity and made sure that all requirements of a scoping review are covered in the protocol. PP contributed to shaping up the idea of the scoping review. She suggested the idea of conducting the review in SAR. Also, she shared her expert opinion on overall conduct of the study. LL contributed to selection of database inclusion for the review. Also, she did major editing on the initial draft of this protocol. She will also contribute to the literature review process.

Funding The authors have not declared a specific grant for this research from any funding agency in the public, commercial or not-for-profit sectors.

Competing interests None declared.

Patient consent for publication Not required.

Provenance and peer review Not commissioned; externally peer reviewed.

Supplemental material This content has been supplied by the author(s). It has not been vetted by BMJ Publishing Group Limited (BMJ) and may not have been peer-reviewed. Any opinions or recommendations discussed are solely those of the author(s) and are not endorsed by BMJ. BMJ disclaims all liability and responsibility arising from any reliance placed on the content. Where the content includes any translated material, BMJ does not warrant the accuracy and reliability of the translations (including but not limited to local regulations, clinical guidelines, terminology, drug names and drug dosages), and is not responsible for any error and/or omissions arising from translation and adaptation or otherwise.

Open access This is an open access article distributed in accordance with the Creative Commons Attribution Non Commercial (CC BY-NC 4.0) license, which permits others to distribute, remix, adapt, build upon this work non-commercially, and license their derivative works on different terms, provided the original work is properly cited, appropriate credit is given, any changes made indicated, and the use is non-commercial. See: http://creativecommons.org/licenses/by-nc/4.0/.

\section{ORCID iD}

Sajid Iqbal http://orcid.org/0000-0003-4358-1178

\section{REFERENCES}

1 World Health Organization. WHO report on the global tobacco epidemic: monitoring tobacco use and prevention policies. 2017: Geneva, Switzerland, 2017. Available: https://apps.who.int/iris/ bitstream/handle/10665/255874/9789241512824-eng.pdf

2 World Health Organization. WHO report on the global tobacco epidemic 2015: raising taxes on tobacco. Geneva, Switzerland, 2015. Available: https://books.google.com.pk/books?hl=en\&lr=\& $i d=3 k 80 D g A A Q B A J \& o i=$ fnd\&pg $=$ PP $1 \& d q=W H O+$ Report + on+the + Global+Tobacco+Epidemic +2015 \&ots=Ec24P85Hsv\&sig=i2unmhC1 VS2YygJO9s2Dcy14SbU\#v=onepage\&q=WHO\%20Report\%20on\% 20the\%20Global\%20Tobacco\%20Epidemic\%202015\&f=false

3 Drope J, Schluger N, Cahn Z. The tobacco atlas. Atlanta: American Cancer Society and Vital Strategies, 2018. https://tobaccoatlas. org/wpcontent/uploads/2018/03/TobaccoAtlas_6thEdition_LoRes_ Rev0318.pdf 
4 Shah N, Siddiqui S. An overview of smoking practices in Pakistan. Pak J Med Sci 2015;31:467.

5 Action on Smoking and Health. Smoking statistics: illness and death. London: ASH, 2016. http://www.ash.org.uk/files/documents/ASH 107.pdf

6 Jha P, Ramasundarahettige C, Landsman V, et al. 21St-Century hazards of smoking and benefits of cessation in the United States. $N$ Engl J Med Overseas Ed 2013;368:341-50

7 National Health Survey. What are the health risks of smoking? 2018. Available: https://www.nhs.uk/common-health-questions/lifestyle/ what-are-the-health-risks-of-smoking/

8 Rigotti NA, Rennard SI, Daughton DM. Benefits and risks of smoking cessation. UpToDate. Waltham (MA): UpToDate, 2014: 357-63. http://www. uptodate.com/contents/benefits-and-risks-of-smokingcessation

9 Rao S, Aslam SK, Zaheer S, et al. Anti-Smoking initiatives and current smoking among 19,643 adolescents in South Asia: findings from the global youth tobacco survey. Harm Reduct J 2014;11:8.

10 Parrota JA, Trosper RL. Traditional forest-related knowledge: sustaining communities, ecosystems and biocultural diversity [book on the internet]. Chapter 9: South Asia. New York: Springer, 2012: 315-56.

11 Sreeramareddy CT, Pradhan PMS, Mir IA, et al. Smoking and smokeless tobacco use in nine South and Southeast Asian countries: prevalence estimates and social determinants from demographic and health surveys. Popul Health Metr 2014;12:22.

12 Mukherjea A, Morgan PA, Snowden LR, et al. Social and cultural influences on tobacco-related health disparities among South Asians in the USA. Tob Control 2012;21:422-8.

13 GBD 2015 Tobacco Collaborators. Smoking prevalence and attributable disease burden in 195 countries and territories, 19902015: a systematic analysis from the global burden of disease study 2015. Lancet 2017;389:1885-906.

14 Borland R, Cooper J, McNeill A, et al. Trends in beliefs about the harmfulness and use of stop-smoking medications and smokeless tobacco products among cigarettes smokers: findings from the ITC four-country survey. Harm Reduct J 2011;8:21.

15 Irfan M, Haque AS, Shahzad H, et al. Reasons for failure to quit: a cross-sectional survey of tobacco use in major cities in Pakistan. Int J Tuberc Lung Dis 2016;20:673-8.

16 Lando HA. Promoting tobacco cessation in low- and middle-income countries. J Smok Cessat 2016;11:66-9.
17 World Health Organization, Research for International Tobacco Control. Who report on the global tobacco epidemic, 2008: the MPOWER package, world Health organization, 2008. Available: https://www.who.int/tobacco/mpower/mpower_report_full_2008. pdf

18 McKay AJ, Patel RKK, Majeed A. Strategies for tobacco control in India: a systematic review. PLoS One 2015;10:e0122610.

19 Jawad M, Jawad S, Waziry RK, et al. Interventions for waterpipe tobacco smoking prevention and cessation: a systematic review. Sci Rep 2016;6:1-8.

20 Arksey H, O'Malley L. Scoping studies: towards a methodological framework. Int J Soc Res Methodol 2005;8:19-32.

21 Munn Z, Peters MDJ, Stern C, et al. Systematic review or scoping review? guidance for authors when choosing between a systematic or scoping review approach. BMC Med Res Methodol 2018;18:143.

22 Bottorff JL, Haines-Saah R, Kelly MT, et al. Gender, smoking and tobacco reduction and cessation: a scoping review. Int J Equity Health 2014;13:1-5.

23 Tricco AC, Lillie E, Zarin W, et al. PRISMA extension for scoping reviews (PRISMA-ScR): checklist and explanation. Ann Intern Med 2018;169:467-73.

24 Peters MDJ, Godfrey C, Mclnerney P. Chapter 11: scoping reviews (2020 version). In: Aromataris E, Munn Z, eds. JBI manual for evidence synthesis, JBI. Adelaide: JBI, 2020. https:// synthesismanual.jbi.global doi.org/

25 Moher D, Shamseer L, Clarke M, et al. Preferred reporting items for systematic review and meta-analysis protocols (PRISMA-P) 2015 statement. Syst Rev 2015;4:1.

26 Levac D, Colquhoun H, O'Brien KK. Scoping studies: advancing the methodology. Implement Sci 2010;5:69.

27 Bramer WM, Rethlefsen ML, Kleijnen J, et al. Optimal database combinations for literature searches in systematic reviews: a prospective exploratory study. Syst Rev 2017;6:245.

28 Jolley RJ, Lorenzetti DL, Manalili K, et al. Protocol for a scoping review study to identify and classify patient-centred quality indicators. BMJ Open 2017;7:e013632.

29 World Health Organization. WHO framework convention on tobacco control. Geneva, Switzerland, 2005. Available: https://apps.who.int/ iris/bitstream/handle/10665/42811/9241591013.pdf?sequence $=1$

30 Ahern KJ. Ten tips for reflexive Bracketing. Qual Health Res 1999:9:407-11. 